\title{
Asymptotic Formulae of the Modal Acoustic Impedance for the Asymmetric Vibrations of a Clamped Circular Plate
}

\author{
W.P. RdzaneK*, W.J. RdzaneK and K. Szemela \\ Department of Acoustics, Institute of Physics, University of Rzeszów \\ al. Rejtana 16A, 35-310 Rzeszów, Poland
}

\begin{abstract}
The asymptotic and approximate formulae for the asymmetric modal acoustic self- and mutual-impedance have been presented for a clamped circular plate embedded into a flat rigid baffle. The formulae have been obtained for the wide frequency band covering the low frequencies, the high frequencies and the middle frequencies. The high frequency asymptotics have been achieved using the method of contour integral and the method of stationary phase. The products of the Bessel and Neumann functions have been expressed as the asymptotic expansions. Further, the approximate formulae valid within the low and middle frequencies have been obtained from the high frequency asymptotics using some mathematical manipulations. The formulae presented are valid for both the axisymmetric vibrations and the asymmetric vibrations.
\end{abstract}

PACS numbers: 43.20.Ks, 43.20.Rz, 43.40.+s

\section{Introduction}

The surface of some structural elements is often excited for vibrations and becomes a source of acoustic waves. The excitation is very often asymmetric in the real vibrating systems and it is very useful to find some asymptotic and approximate formulae for the modal quantities necessary to compute the acoustic power, the acoustic pressure and the structure's vibration velocity including the fluid loading. So far, a number of such formulae have been presented for vibrating circular and rectangular membranes and plates using several approximate methods [1-9]. The results obtained by using the exact solutions for the free vibrations have been presented in a few studies [10-12]. The corresponding asymptotic formulae for the acoustic impedance and the acoustic power have been reported in [13-17]. However, the formulae are appropriate for the axisymmetric processes only. Therefore, this paper focuses on the asymptotics and the approximations useful for the asymmetric vibroacoustic quantities of the vibrating clamped circular plate valid within almost the whole frequency range, i.e. for the high frequencies, the low frequencies and the middle frequencies, except the very low frequencies and the frequencies close to the plate's eigenfrequencies.

\section{Asymptotic formulae for the modal acoustic impedance}

The modal impedance has no pure physical interpretation. This quantity together with the coupling ma-

\footnotetext{
* corresponding author; e-mail: wprdzank@univ.rzeszow.pl
}

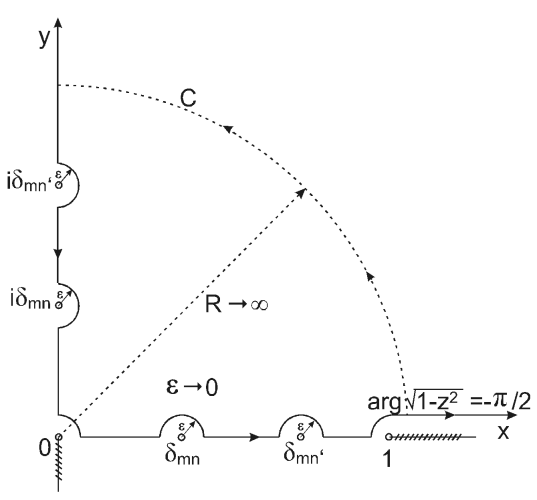

Fig. 1. The integration path $C$ used to derive the asymptotics for the mutual resistance.

trix and the given excitation provides the total acoustic impedance. This was reported by Morse and Ingard for the acoustic wave transmission through a circular membrane in considerable detail in $[16,18]$ and also for the acoustic power radiated into the quarter space by the membrane in [13]. The connection of the modal acoustic impedance and the coupling matrix has been described in Appendix B for the reader's convenience. However, this paper focuses only on the modal acoustic impedance.

The integral formulae for the modal acoustic impedance of the two different vibrating clamped circular plates embedded into a flat rigid baffle have been presented earlier in [17]. The formulae can be simplified to be valid for a single plate and the modal self impedance is 


$$
\begin{aligned}
& \zeta_{m ; n}=\theta_{m ; n}-i \chi_{m ; n}, \\
& \theta_{m ; n}=4 \delta_{m n}^{4} \int_{0}^{1} \frac{\left[\alpha_{m n} \delta_{m n} J_{m}(\beta x)-x J_{m+1}(\beta x)\right]^{2} x \mathrm{~d} x}{\left(x^{4}-\delta_{m n}^{4}\right)^{2} \sqrt{1-x^{2}}}, \\
& \chi_{m ; n}=4 \delta_{m n}^{4} \int_{1}^{\infty} \frac{\left[\alpha_{m n} \delta_{m n} J_{m}(\beta x)-x J_{m+1}(\beta x)\right]^{2} x \mathrm{~d} x}{\left(x^{4}-\delta_{m n}^{4}\right)^{2} \sqrt{x^{2}-1}},
\end{aligned}
$$

where it has been denoted

$$
\alpha_{m n}=J_{m+1}\left(\beta_{m n}\right) / J_{m}\left(\beta_{m n}\right), \quad \delta_{m n}=\beta_{m n} / \beta, \quad \beta=k_{0} a,
$$

$J_{m}(\cdot)$ is the Bessel function of the $m$ th order, $a$ is the radius of the plate, $k_{0}$ is the acoustic wavenumber, $\beta_{m n}$ is the eigenvalue, and $i^{2}=-1$. The frequency equation is $J_{m}\left(\beta_{m n}\right) I_{m+1}\left(\beta_{m n}\right)+J_{m+1}\left(\beta_{m n}\right) I_{m}\left(\beta_{m n}\right)=0$ where $I_{m}(\cdot)$ is the modified Bessel function of the $m$ th order. The modal mutual impedance assumes the form of

$$
\begin{aligned}
& \zeta_{m ; n, n^{\prime}}=\theta_{m ; n, n^{\prime}}-i \chi_{m ; n, n^{\prime}}, \\
& \theta_{m ; n, n^{\prime}}=4 \delta_{m n}^{2} \delta_{m n^{\prime}}^{2} \int_{0}^{1} \frac{\Psi_{m ; n, n^{\prime}}(x) x \mathrm{~d} x}{\left(x^{4}-\delta_{m n}^{4}\right)\left(x^{4}-\delta_{m n^{\prime}}^{4}\right) \sqrt{1-x^{2}}}, \\
& \chi_{m ; n, n^{\prime}}=4 \delta_{m n}^{2} \delta_{m n^{\prime}}^{2} \int_{1}^{\infty} \frac{\Psi_{m ; n, n^{\prime}}(x) x \mathrm{~d} x}{\left(x^{4}-\delta_{m n}^{4}\right)\left(x^{4}-\delta_{m n^{\prime}}^{4}\right) \sqrt{x^{2}-1}}, \\
& \Psi_{m ; n, n^{\prime}}(x)=\left(\alpha_{m n} \delta_{m n} J_{m}(\beta x)-x J_{m+1}(\beta x)\right)\left(\alpha_{m n^{\prime}} \delta_{m n^{\prime}} J_{m}(\beta x)-x J_{m+1}(\beta x)\right)
\end{aligned}
$$

This modal quantity is necessary for further calculations of the vibroacoustic properties of the acoustic system under consideration. Using Eqs. (1) and (3) makes it possible to calculate the modal impedance of the vibrating circular plate for the whole frequency spectrum. However, their integral form causes numerical problems. Therefore, a sort of asymptotic and approximate formulae has been developed and reported below to be useful for some further numerical calculations.

\subsection{The modal self-impedance within the high frequencies}

The following condition $\delta_{m n}<1$ is satisfied for the self-impedance within the high frequency range. The asymptotic formulation for the resistance can be obtained by using the method of path integral analysis. For this purpose, the following complex variable function has been introduced $[10,16,17]$

$$
F^{(s)}(z)=\alpha_{m n}^{2} \delta_{m n}^{2} J_{m}(\beta z) H_{m}^{(1)}(\beta z)-2 \alpha_{m n} \delta_{m n} z J_{m}(\beta z) H_{m+1}^{(1)}(\beta z)+z^{2} J_{m+1}(\beta z) H_{m+1}^{(1)}(\beta z),
$$

where $H_{m}^{(1)}(\cdot)=J_{m}(\cdot)+i N_{m}(\cdot)$ is the Hankel function of the first kind and $m$ th order, $N_{m}(\cdot)$ denotes the Neumann function of the $m$ th order. Additionally, the following relation is satisfied

$$
\operatorname{Re} F^{(s)}(x)=\alpha_{m n}^{2} \delta_{m n}^{2} J_{m}^{2}(\beta x)-2 \alpha_{m n} \delta_{m n} x J_{m}(\beta x) J_{m+1}(\beta x)+x^{2} J_{m+1}^{2}(\beta x)
$$

The integration path has been chosen according to Fig. 1 with this difference that there are only two second order poles at points $z=\delta_{m n}$ and $z=i \delta_{m n}$. Then applying the Cauchy theorem leads to

$$
\oint_{\mathrm{C}} \frac{F^{(s)}(z) z \mathrm{~d} z}{\left(z^{4}-\delta_{m n}^{4}\right)^{2} \sqrt{1-z^{2}}}=0,
$$

where the integrand is analytical and regular on the contour $C$ and within its interior. Performing integration along the big circle for $R \rightarrow \infty$ gives no contribution. Since $F^{(s)}(i y)=0$ for $y \in R$, the following asymptotic formula for the modal acoustic resistance has been formulated as [17]

$$
\theta_{m n}^{\text {(high) }}=4 \delta_{m n}^{4}\left[\int_{1}^{\infty} \frac{\operatorname{Im} F^{(s)}(x) x \mathrm{~d} x}{\left(x^{4}-\delta_{m n}^{4}\right)^{2} \sqrt{x^{2}-1}}+\Lambda\left(\delta_{m n}\right)\right],
$$

where

$$
\begin{aligned}
& \Lambda\left(\delta_{m n}\right)=\frac{1}{4 \delta_{m n}^{4}}\left[\frac{1+\alpha_{m n}^{2}}{2 \sqrt{1-\delta_{m n}^{2}}}+\frac{1-\alpha_{m n}^{2}}{2 \sqrt{1+\delta_{m n}^{2}}}\right] \\
& \quad+\frac{m \alpha_{m n}}{4 \delta_{m n}^{4} \beta_{m n}}\left[\frac{1}{\sqrt{1+\delta_{m n}^{2}}}-\frac{1}{\sqrt{1-\delta_{m n}^{2}}}\right]-\frac{\alpha_{m n}}{8 \beta \delta_{m n}^{5}}\left[\frac{3 \delta_{m n}^{2}-2}{\left(1-\delta_{m n}^{2}\right)^{3 / 2}}+\frac{3 \delta_{m n}^{2}+2}{\left(1+\delta_{m n}^{2}\right)^{3 / 2}}\right]
\end{aligned}
$$

is the real component of the sum of residues (cf. [10]). The upper indice "(high)" denotes that the asymptotic expression is valid for the high frequencies. Taking into consideration that

$$
\operatorname{Im} F^{(s)}(x)=\alpha_{m n}^{2} \delta_{m n}^{2} J_{m}(\beta x) N_{m}(\beta x)-2 \alpha_{m n} \delta_{m n} x J_{m}(\beta x) N_{m+1}(\beta x)+x^{2} J_{m+1}(\beta x) N_{m+1}(\beta x)
$$

the integral from Eq. (6) can be formulated as 


$$
\int_{1}^{\infty} \frac{\operatorname{Im} F^{(s)}(x) x \mathrm{~d} x}{\left(x^{4}-\delta_{m n}^{4}\right)^{2} \sqrt{x^{2}-1}}=\alpha_{m n}^{2} \delta_{m n}^{2} A_{a}^{(s)}-2 \alpha_{m n} \delta_{m n} B_{a}^{(s)}+C_{a}^{(s)},
$$

where

$$
\begin{aligned}
& A_{a}^{(s)}=\int_{1}^{\infty} \frac{J_{m}(\beta x) N_{m}(\beta x) x \mathrm{~d} x}{\left(x^{4}-\delta_{m n}^{4}\right)^{2} \sqrt{x^{2}-1}}, \\
& B_{a}^{(s)}=\int_{1}^{\infty} \frac{J_{m}(\beta x) N_{m+1}(\beta x) x^{2} \mathrm{~d} x}{\left(x^{4}-\delta_{m n}^{4}\right)^{2} \sqrt{x^{2}-1}}, \\
& C_{a}^{(s)}=\int_{1}^{\infty} \frac{J_{m+1}(\beta x) N_{m+1}(\beta x) x^{3} \mathrm{~d} x}{\left(x^{4}-\delta_{m n}^{4}\right)^{2} \sqrt{x^{2}-1}} .
\end{aligned}
$$

The Bessel and Neumann functions can be presented by means of the following expansion series [16, 20]

$$
\begin{aligned}
& J_{m}(\beta x)=\sqrt{\frac{2}{\pi \beta x}} \sum_{j=0}^{\infty}\left[\frac{g_{m}(j)}{x^{2 j}} \cos \left(\varphi_{m}\right)-\frac{h_{m}(j)}{x^{2 j+1}} \sin \left(\varphi_{m}\right)\right], \\
& N_{m}(\beta x)=\sqrt{\frac{2}{\pi \beta x}} \sum_{j=0}^{\infty}\left[\frac{g_{m}(j)}{x^{2 j}} \sin \left(\varphi_{m}\right)+\frac{h_{m}(j)}{x^{2 j+1}} \cos \left(\varphi_{m}\right)\right],
\end{aligned}
$$

where

$$
\begin{aligned}
& g_{m}(j)=\frac{(-1)^{j}}{(2 j) !(8 \beta)^{2 j}} \prod_{k=0}^{2 j-1}\left[(2 m)^{2}-(2 k+1)^{2}\right], \\
& h_{m}(j)=\frac{(-1)^{j}}{(2 j+1) !(8 \beta)^{2 j+1}} \prod_{k=0}^{2 j}\left[(2 m)^{2}-(2 k+1)^{2}\right], \\
& \varphi_{m}=\beta x-\pi / 4-m \pi / 2
\end{aligned}
$$

From practical viewpoint, it is necessary to limit the expansion series to a finite number of terms. In order to take into account only first $N$ terms for calculations it has been assumed that the upper limit of sums are equal to $N-1$. On the basis of the above formulae, the products of special functions appearing in Eq. (9) can be presented as the following expansion series

$$
\begin{aligned}
& J_{m}(\beta x) N_{m}(\beta x) \approx \frac{(-1)^{m}}{\pi \beta x} \sum_{r=0}^{N-1} \sum_{s=0}^{N-1}\left[-\frac{g_{m}(r) g_{m}(s)}{x^{2 r+2 s}} \cos 2 \beta x\right. \\
& +\frac{\hat{\nu}_{m, m}(s, r)}{x^{2 s+2 r+1}} \sin 2 \beta x+\frac{h_{m}(r) h_{m}(s)}{x^{2 r+2 s+2}} \cos 2 \beta x(-1)^{m}+\frac{\hat{\gamma}_{m, m}(r, s)}{\left.x^{2 r+2 s+1}\right]} \\
& J_{m}(\beta x) N_{m+1}(\beta x) \approx \frac{(-1)^{m}}{\pi \beta x} \sum_{r=0}^{N-1} \sum_{s=0}^{N-1}\left[-\frac{g_{m}(r) g_{m+1}(s)}{x^{2 r+2 s}} \sin 2 \beta x-\frac{\hat{\nu}_{m, m+1}(r, s)}{x^{2 s+2 r+1}} \cos 2 \beta x\right. \\
& \left.+\frac{h_{m}(r) h_{m+1}(s)}{x^{2 r+2 s+2}} \sin 2 \beta x-(-1)^{m} \frac{g_{m}(r) g_{m+1}(s)}{x^{2 s+2 r}}-(-1)^{m} \frac{h_{m}(r) h_{m+1}(s)}{x^{2 s+2 r+2}}\right],
\end{aligned}
$$

where

$$
\hat{\nu}_{m, k}(r, s)=g_{m}(r) h_{k}(s)+g_{k}(s) h_{m}(r), \quad \hat{\gamma}_{m, k}(r, s)=g_{m}(r) h_{k}(s)-g_{k}(s) h_{m}(r) .
$$

The asymptotics for integrals in Eq. (9) can be derived after employing expansion series from Eq. (12) and carrying out integration term by term. The following formula has been obtained by means of the stationary phase method $[10,16,17]$

$$
\int_{1}^{\infty} \frac{\exp (i 2 \beta x) x^{r} \mathrm{~d} x}{\left(x^{4}-\delta_{m n}^{4}\right)^{2} \sqrt{x^{2}-1}} \approx \frac{1}{2} \sqrt{\frac{\pi}{\beta}} \frac{\exp (2 \beta+\pi / 4)}{\left(1-\delta_{m n}^{4}\right)^{2}},
$$

for any $r$ which is useful for further integration. After integration in Eq. (9), the asymptotic expression for the acoustic self-resistance has been formulated in the form where the oscillating and non-oscillating components are separated

$$
\theta_{m ; n}^{(\text {high })}=\tilde{\theta}_{m ; n}+\bar{\theta}_{m ; n}^{(\text {high })} .
$$

The oscillating and non-oscillating components have been denoted by the symbols " " and "-", respectively. The asymptotic oscillating component can be formulated as

$$
\tilde{\theta}_{m ; n}=\frac{2(-1)^{m} \delta_{m n}^{4}}{\sqrt{\pi} \beta^{3 / 2}\left(1-\delta_{m n}^{4}\right)^{2}}\left[X_{a}^{(s)} \cos (2 \beta+\pi / 4)+Y_{a}^{(s)} \sin (2 \beta+\pi / 4)\right],
$$


where

$$
\begin{aligned}
& X_{a}^{(s)}=\alpha_{m n}^{2} \delta_{m n}^{2} u_{m, m}(\beta)+2 \alpha_{m n} \delta_{m n} \nu_{m, m+1}(\beta)-u_{m+1, m+1}(\beta), \\
& Y_{a}^{(s)}=\alpha_{m n}^{2} \delta_{m n}^{2} \nu_{m, m}(\beta)-2 \alpha_{m n} \delta_{m n} u_{m, m+1}(\beta)-\nu_{m+1, m+1}(\beta), \\
& u_{m, k}(\beta)=\sum_{r=0}^{N-1} \sum_{s=0}^{N-1}\left[h_{m}(r) h_{k}(s)-g_{m}(r) g_{k}(s)\right], \\
& \nu_{m, k}(\beta)=\sum_{r=0}^{N-1} \sum_{s=0}^{N-1} \hat{\nu}_{m, k}(s, r) .
\end{aligned}
$$

The following asymptotic formulation has been achieved

$$
\bar{\theta}_{m ; n}^{(\text {high })}=4 \delta_{m n}^{4}\left[\frac{1}{\pi \beta} \sum_{r=0}^{N-1} \sum_{s=0}^{N-1}\left(\Omega^{(s)}(r, s) I_{2 r+2 s+1}^{(s)}\left(\delta_{m n}\right)+\lambda^{(s)}(r, s) I_{2 r+2 s-1}^{(s)}\left(\delta_{m n}\right)\right)+\Lambda\left(\delta_{m n}\right)\right],
$$

for the non-oscillating component where

$$
\begin{aligned}
& \Omega^{(s)}(r, s)=\alpha_{m n}^{2} \delta_{m n}^{2} \hat{\gamma}_{m, m}(r, s)+2 \alpha_{m n} \delta_{m n} h_{m}(r) h_{m+1}(s), \\
& \lambda^{(s)}(r, s)=\hat{\gamma}_{m, m+1}(r, s)+2 \alpha_{m n} \delta_{m n} g_{m}(r) g_{m+1}(s), \\
& I_{n}^{(s)}(b)=\int_{1}^{\infty} \frac{\mathrm{d} x}{x^{n}\left(x^{4}-b^{4}\right)^{2} \sqrt{x^{2}-1}} .
\end{aligned}
$$

Employing Eqs. (A.5) gives the non-oscillating component of the modal self-resistance in the form covering only the elementary functions

$$
\bar{\theta}_{m ; n}^{(\text {high })}=4 \delta_{m n}^{4}\left[U_{a}^{(s)}+\frac{V_{a}^{(s)}}{\sqrt{1-\delta_{m n}^{2}}}+\Lambda\left(\delta_{m n}\right)\right],
$$

where

$$
\begin{aligned}
& U_{a}^{(s)}=\frac{1}{\pi \beta} \sum_{r=0}^{N-1} \sum_{s=0}^{N-1}\left(\Omega^{(s)} a_{2 r+2 s+1}^{(s)}\left(\delta_{m n}\right)+\lambda^{(s)} a_{2 r+2 s-1}^{(s)}\left(\delta_{m n}\right)\right), \\
& V_{a}^{(s)}=\frac{1}{\beta} \sum_{r=0}^{N-1} \sum_{s=0}^{N-1}\left(\Omega^{(s)} b_{2 r+2 s+1}^{(s)}\left(\delta_{m n}\right)+\lambda^{(s)} b_{2 r+2 s-1}^{(s)}\left(\delta_{m n}\right)\right)
\end{aligned}
$$

and the functions $a_{n}^{(s)}(b)$ and $b_{n}^{(s)}(b)$ have been defined in App. A.

On the basis of Eq. (1), the modal self-reactance has been formulated as

$$
\chi_{m ; n}=4 \delta_{m n}^{4}\left[\alpha_{m n}^{2} \delta_{m n}^{2} A_{p}^{(s)}-2 \alpha_{m n} \delta_{m n} B_{p}^{(s)}+C_{p}^{(s)}\right],
$$

where

$$
\begin{aligned}
& A_{p}^{(s)}=\int_{1}^{\infty} \frac{J_{m}^{2}(\beta x) x \mathrm{~d} x}{\left(x^{4}-\delta_{m n}^{4}\right)^{2} \sqrt{x^{2}-1}}, \quad B_{p}^{(s)}=\int_{1}^{\infty} \frac{J_{m}(\beta x) J_{m+1}(\beta x) x^{2} \mathrm{~d} x}{\left(x^{4}-\delta_{m n}^{4}\right)^{2} \sqrt{x^{2}-1}}, \\
& C_{p}^{(s)}=\int_{1}^{\infty} \frac{J_{m+1}^{2}(\beta x) x^{3} \mathrm{~d} x}{\left(x^{4}-\delta_{m n}^{4}\right)^{2} \sqrt{x^{2}-1}} .
\end{aligned}
$$

Making use of Eqs. (10) leads to

$$
\begin{aligned}
& J_{m}^{2}(\beta x) \approx \frac{(-1)^{m}}{\pi \beta x} \sum_{r=0}^{N-1} \sum_{s=0}^{N-1}\left[\frac{g_{m}(r) g_{m}(s)}{x^{2 r+2 s}} \sin 2 \beta x+\frac{\hat{\nu}_{m, m}(r, s)}{x^{2 r+2 s+1}} \cos 2 \beta x-\frac{h_{m}(r) h_{m}(s)}{x^{2 r+2 s+2}} \sin 2 \beta x\right. \\
& \left.+(-1)^{m} \frac{g_{m}(r) g_{m}(s)}{x^{2 r+2 s}}+(-1)^{m} \frac{h_{m}(r) h_{m}(s)}{x^{2 r+2 s+2}},\right] \\
& J_{m}(\beta x) J_{m+1}(\beta x) \approx \frac{(-1)^{m}}{\pi \beta x} \sum_{r=0}^{N-1} \sum_{s=0}^{N-1}\left[-\frac{g_{m}(r) g_{m+1}(s)}{x^{2 r+2 s}} \cos 2 \beta x+\frac{\hat{\nu}_{m, m+1}(r, s)}{x^{2 r+2 s+1}} \sin 2 \beta x\right. \\
& \left.+\frac{h_{m}(r) h_{m+1}(s)}{x^{2 r+2 s+2}} \cos 2 \beta x+(-1)^{m} \frac{\hat{\gamma}_{m, m+1}(r, s)}{x^{2 r+2 s+1}}\right] .
\end{aligned}
$$


Integrals given in Eq. (23) have been calculated by using of the above expansion series and Eq. (14). Further, the asymptotic modal self-reactance has been formulated as the sum of the oscillating and non-oscillating components

$$
\chi_{m ; n}^{(\mathrm{high})}=\tilde{\chi}_{m ; n}+\bar{\chi}_{m ; n}^{(\mathrm{high})}
$$

by using Eq. (22). The oscillating component assumes the form of

$$
\tilde{\chi}_{m ; n}=\frac{2(-1)^{m} \delta_{m n}^{4}}{\sqrt{\pi} \beta^{3 / 2}\left(1-\delta_{m n}^{4}\right)^{2}}\left[X_{p}^{(s)} \cos (2 \beta+\pi / 4)+Y_{p}^{(s)} \sin (2 \beta+\pi / 4)\right],
$$

where

$$
\begin{aligned}
& X_{p}^{(s)}=\alpha_{m n}^{2} \delta_{m n}^{2} \nu_{m, m}(\beta)-2 \alpha_{m n} \delta_{m n} u_{m, m+1}(\beta)-\nu_{m+1, m+1}(\beta), \\
& Y_{p}^{(s)}=-\alpha_{m n}^{2} \delta_{m n}^{2} u_{m, m}(\beta)-2 \alpha_{m n} \delta_{m n} \nu_{m, m+1}(\beta)+u_{m+1, m+1}(\beta),
\end{aligned}
$$

whereas the non-oscillating component is

$$
\begin{aligned}
& \bar{\chi}_{m ; n}^{\text {(high })}=\frac{4 \delta_{m n}^{4}}{\pi \beta} \sum_{r=0}^{\infty} \sum_{s=0}^{\infty}\left[\hat{\phi}^{(s)}(r, s) I_{2 r+2 s}^{(s)}\left(\delta_{m n}\right)+\alpha_{m n}^{2} \delta_{m n}^{2} h_{m}(r) h_{m}(s) I_{2 r+2 s+2}^{(s)}\left(\delta_{m n}\right)\right. \\
& \left.\quad+g_{m+1}(r) g_{m+1}(s) I_{2 r+2 s-2}^{(s)}\left(\delta_{m n}\right)\right],
\end{aligned}
$$

where

$$
\hat{\phi}^{(s)}(r, s)=\alpha_{m n}^{2} \delta_{m n}^{2} g_{m}(r) g_{m}(s)-2 \alpha_{m n} \delta_{m n} \hat{\gamma}_{m, m+1}(r, s)+h_{m+1}(r) h_{m+1}(s) .
$$

Applying Eqs. (A.5) gives

$$
\bar{\chi}_{m ; n}^{(\text {high })}=4 \delta_{m n}^{4}\left[U_{p}^{(s)}+\frac{V_{p}^{(s)}}{\sqrt{1-\delta_{m n}^{2}}} \arcsin \delta_{m n}\right],
$$

where

$$
\begin{aligned}
& U_{p}^{(s)}=\frac{1}{\pi \beta} \sum_{r=0}^{N-1} \sum_{s=0}^{N-1}\left[\hat{\phi}^{(s)}(r, s) a_{2 r+2 s}^{(s)}\left(\delta_{m n}\right)+\alpha_{m n}^{2} \delta_{m n}^{2} h_{m}(r) h_{m}(s) a_{2 r+2 s+2}^{(s)}\left(\delta_{m n}\right)+g_{m+1}(r) g_{m+1}(s) a_{2 r+2 s-2}^{(s)}\left(\delta_{m n}\right)\right], \\
& V_{p}^{(s)}=\frac{1}{\pi \beta} \sum_{r=0}^{N-1} \sum_{s=0}^{N-1}\left[\hat{\phi}^{(s)}(r, s) b_{2 r+2 s}^{(s)}\left(\delta_{m n}\right)+\alpha_{m n}^{2} \delta_{m n}^{2} h_{m}(r) h_{m}(s) b_{2 r+2 s+2}^{(s)}\left(\delta_{m n}\right)+g_{m+1}(r) g_{m+1}(s) b_{2 r+2 s-2}^{(s)}\left(\delta_{m n}\right)\right] .
\end{aligned}
$$

The absolute correction to the true value connected with the asymptotic formulae (16), (20), (26) and (30) can be estimated for the high frequencies as [16]

$$
\left|\Delta \theta_{m ; n}^{(\text {high })}\right|,\left|\Delta \chi_{m ; n}^{(\text {high })}\right| \leq \delta_{m n}^{4} \beta^{-3 / 2}=E_{t}^{(\text {high })},
$$

where the true value of considered quantities means that the corresponding value has been calculated using the integral formulae. Moreover, it has been assumed that the numerical integrations do not produce any numerical errors. The absolute correction decreases rapidly with an increase in the parameter $\beta$ and the vibration frequency associated. The asymptotics obtained can be useful for some further numerical calculations of the self-impedance for the high frequencies.

\subsection{The modal self-impedance within the low frequencies}

The approximate formulae presented in the previous section in Eqs. (20) and (30) can be transformed using the following equations [19]

$$
\arcsin x=\frac{\pi}{2}-i \operatorname{arccosh} x, \quad \sqrt{1-x^{2}}=i \sqrt{x^{2}-1} \text { for } x>1
$$

to be valid within the low frequency range, i.e. for $\delta_{m n}>1[16,19]$. In this case they can be formulated as follows

$$
\begin{aligned}
& \theta_{m ; n}^{(\text {low })}=\tilde{\theta}_{m ; n}+\bar{\theta}_{m ; n}^{(\text {low })}, \quad \chi_{m ; n}^{(\text {low })}=\tilde{\chi}_{m ; n}+\bar{\chi}_{m ; n}^{(\text {low })} \\
& \bar{\theta}_{m ; n}^{(\text {low })}=4 \delta_{m n}^{4}\left[\frac{1-\alpha_{m n}^{2}}{8 \delta_{m n}^{4} \sqrt{1+\delta_{m n}^{2}}}+\frac{m \alpha_{m n}}{4 \delta_{m n}^{4} \beta_{m n} \sqrt{1+\delta_{m n}^{2}}}+U_{a}^{(s)}-\frac{\alpha_{m n}\left(3 \delta_{m n}^{2}+2\right)}{8 \beta \delta_{m n}^{5}\left(1+\delta_{m n}^{2}\right)^{3 / 2}}-\frac{\pi V_{p}^{(s)}}{2 \sqrt{\delta_{m n}^{2}-1}}\right], \\
& \bar{\chi}_{m ; n}^{(\text {low })}=4 \delta_{m n}^{4}\left[\frac{1+\alpha_{m n}^{2}}{8 \delta_{m n}^{4} \sqrt{\delta_{m n}^{2}-1}}-\frac{m \alpha_{m n}}{4 \delta_{m n}^{4} \beta_{m n} \sqrt{\delta_{m n}^{2}-1}}+U_{p}^{(s)}+\frac{V_{a}^{(s)}}{\sqrt{\delta_{m n}^{2}-1}}+\frac{\alpha_{m n}\left(3 \delta_{m n}^{2}-2\right)}{8 \beta \delta_{m n}^{5}\left(\delta_{m n}^{2}-1\right)^{3 / 2}}\right. \\
& \left.-\frac{V_{p}^{(s)} \arccos h \delta_{m n}}{\sqrt{\delta_{m n}^{2}-1}}\right] .
\end{aligned}
$$


It is worth noticing that this rearrangement does not influence the oscillating components of the modal impedance. Only the non-oscillating components are modified. The upper indice "(low)" means that the formulation is valid within the low frequency range. The absolute correction to the true value for formulae representing self-impedance and self-reactance within the low frequency range is after [16]

$$
\left|\Delta \theta_{m ; n}^{(\text {low })}\right|,\left|\Delta \chi_{m ; n}^{(\text {low })}\right| \leq \delta_{m n}^{-4} \beta^{-3 / 2}=E_{t}^{(\text {low })}
$$

The approximate formulae are useful for $7<\beta<\beta_{m n}$. They are useless for $\beta<7$ since they have been obtained using the asymptotic formulae from Eq. (10).

\subsection{The modal mutual impedance within the high frequencies}

The high frequency range in the case of the mutual-impedance is determined by means of the following relation $\delta_{m n}, \delta_{m n^{\prime}}<1$.

Making use of the following identity

$$
\frac{1}{\left(x^{4}-b_{1}^{4}\right)\left(x^{4}-b_{2}^{4}\right)}=\frac{1}{b_{1}^{4}-b_{2}^{4}}\left[\frac{1}{\left(x^{4}-b_{1}^{4}\right)}-\frac{1}{\left(x^{4}-b_{2}^{4}\right)}\right]
$$

it is possible to express the modal mutual impedance from Eq. (3) in the form of (cf. [16])

$$
\begin{aligned}
& \theta_{m ; n, n^{\prime}}=S_{m ; n}-S_{m ; n^{\prime}}, \quad \chi_{m ; n, n^{\prime}}=W_{m ; n}-W_{m ; n^{\prime}}, \\
& S_{m ; n}=\frac{4 \delta_{m n}^{2} \delta_{m n^{\prime}}^{2}}{\delta_{m n}^{4}-\delta_{m n^{\prime}}^{4}} \int_{0}^{1} \frac{\Psi_{m ; n, n^{\prime}}(x) x \mathrm{~d} x}{\left(x^{4}-\delta_{m n}^{4}\right) \sqrt{1-x^{2}}}, \quad S_{m ; n^{\prime}}=\frac{4 \delta_{m n}^{2} \delta_{m n^{\prime}}^{2}}{\delta_{m n}^{4}-\delta_{m n^{\prime}}^{4}} \int_{0}^{1} \frac{\Psi_{m ; n, n^{\prime}}(x) x \mathrm{~d} x}{\left(x^{4}-\delta_{m n^{\prime}}^{4}\right) \sqrt{1-x^{2}}}, \\
& W_{m ; n}=\frac{4 \delta_{m n}^{2} \delta_{m n^{\prime}}^{2}}{\delta_{m n}^{4}-\delta_{m n^{\prime}}^{4}} \int_{1}^{\infty} \frac{\Psi_{m ; n, n^{\prime}}(x) x \mathrm{~d} x}{\left(x^{4}-\delta_{m n}^{4}\right) \sqrt{x^{2}-1}}, \quad W_{m ; n^{\prime}}=\frac{4 \delta_{m n}^{2} \delta_{m n^{\prime}}^{2}}{\delta_{m n}^{4}-\delta_{m n^{\prime}}^{4}} \int_{1}^{\infty} \frac{\Psi_{m ; n, n^{\prime}}(x) x \mathrm{~d} x}{\left(x^{4}-\delta_{m n^{\prime}}^{4}\right) \sqrt{x^{2}-1}} .
\end{aligned}
$$

The asymptotics for the quantity $S_{m ; n}$, appropriate to frequencies above the coincidence, can be obtained in the similar way as in the case of the self-resistance by applying the method of the contour integral. For this purpose, the following function has been introduced

$$
\begin{aligned}
& F^{(m)}(z)=\alpha_{m n} \delta_{m n} \alpha_{m n^{\prime}} \delta_{m n^{\prime}} J_{m}(\beta z) H_{m}^{(1)}(\beta z)-\left(\alpha_{m n} \delta_{m n}+\alpha_{m n^{\prime}} \delta_{m n^{\prime}}\right) z J_{m}(\beta z) H_{m+1}^{(1)}(\beta z) \\
& \quad+z^{2} J_{m+1}(\beta z) H_{m+1}^{(1)}(\beta z)
\end{aligned}
$$

and the following equation has been formulated

$$
\oint_{\mathrm{C}} \frac{F^{(m)} z d z}{\left(z^{4}-\delta_{m n}^{4}\right) \sqrt{1-z^{2}}}=0 .
$$

The integration has been performed along the path shown in Fig. 1 . There are the first order poles at $z=\delta_{m n}$ and $z=i \delta_{m n}$ which results in

$$
S_{m ; n}=\frac{4 \delta_{m n}^{2} \delta_{m n^{\prime}}^{2}}{\delta_{m n}^{4}-\delta_{m n^{\prime}}^{4}}\left[\int_{1}^{\infty} \frac{\operatorname{Im} F^{(m)}(x) x \mathrm{~d} x}{\left(x^{4}-\delta_{m n}^{4}\right) \sqrt{x^{2}-1}}+\frac{\alpha_{m n^{\prime}} \delta_{m n^{\prime}}}{2 \delta_{m n}^{2} \beta}\left[\frac{1}{\sqrt{1+\delta_{m n}^{2}}}-\frac{1}{\sqrt{1-\delta_{m n}^{2}}}\right]\right] .
$$

The integral appearing in the above equation can also be presented in the following form of

$$
\int_{1}^{\infty} \frac{\operatorname{Im} F^{(m)}(x) x \mathrm{~d} x}{\left(x^{4}-\delta_{m n}^{4}\right) \sqrt{x^{2}-1}}=\alpha_{m n} \delta_{m n} \alpha_{m n^{\prime}} \delta_{m n^{\prime}} A_{a}^{(m)}-\left(\alpha_{m n} \delta_{m n}+\alpha_{m n^{\prime}} \delta_{m n^{\prime}}\right) \alpha_{m n} \delta_{m n} B_{a}^{(m)}+C_{a}^{(m)},
$$

where

$$
\begin{aligned}
& A_{a}^{(m)}=\int_{1}^{\infty} \frac{J_{m}(\beta x) N_{m}(\beta x) x \mathrm{~d} x}{\left(x^{4}-\delta_{m n}^{4}\right) \sqrt{x^{2}-1}}, \quad B_{a}^{(m)}=\int_{1}^{\infty} \frac{J_{m}(\beta x) N_{m+1}(\beta x) x^{2} \mathrm{~d} x}{\left(x^{4}-\delta_{m n}^{4}\right) \sqrt{x^{2}-1}}, \\
& C_{a}^{(m)}=\int_{1}^{\infty} \frac{J_{m+1}(\beta x) N_{m+1}(\beta x) x^{3} \mathrm{~d} x}{\left(x^{4}-\delta_{m n}^{4}\right) \sqrt{x^{2}-1}} .
\end{aligned}
$$

Calculations of the integrals in Eq. (42) have been conducted by employing the expansion series from Eq. (12) and making use of the following formula

$$
\int_{1}^{\infty} \frac{\exp (i 2 \beta x) x^{r} \mathrm{~d} x}{\left(x^{4}-\delta_{m n}^{4}\right) \sqrt{x^{2}-1}} \approx \frac{1}{2} \sqrt{\frac{\pi}{\beta}} \frac{\exp (2 \beta+\pi / 4)}{\left(1-\delta_{m n}^{4}\right)}
$$

for any $r$. Finally, the asymptotic formula of the modal acoustic impedance can be expressed as

$$
S_{m ; n}^{(\text {high })}=\tilde{S}_{m ; n}+\bar{S}_{m ; n}^{(\text {high })}
$$

where the oscillating component assumes the form of

$$
\tilde{S}_{m n}=\frac{2(-1)^{m} \delta_{m n}^{2} \delta_{m n^{\prime}}^{2}}{\sqrt{\pi} \beta^{3 / 2}\left(1-\delta_{m n}^{4}\right)\left(\delta_{m n}^{4}-\delta_{m n^{\prime}}^{4}\right)}\left[X_{a}^{(m)} \cos (2 \beta+\pi / 4)+Y_{a}^{(m)} \sin (2 \beta+\pi / 4)\right]
$$

where 


$$
\begin{aligned}
& X_{a}^{(m)}=\alpha_{m n} \delta_{m n} \alpha_{m n^{\prime}} \delta_{m n^{\prime}} u_{m, m}(\beta)+\left(\alpha_{m n} \delta_{m n}+\alpha_{m n^{\prime}} \delta_{m n^{\prime}}\right) \nu_{m, m+1}(\beta)-u_{m+1, m+1}(\beta), \\
& Y_{a}^{(m)}=\alpha_{m n} \delta_{m n} \alpha_{m n^{\prime}} \delta_{m n^{\prime}} \nu_{m, m}(\beta)-\left(\alpha_{m n} \delta_{m n}+\alpha_{m n^{\prime}} \delta_{m n^{\prime}}\right) u_{m, m+1}(\beta)-\nu_{m+1, m+1}(\beta) .
\end{aligned}
$$

By applying Eqs. (A.5) the non-oscillating component of $S_{m ; n}^{(\text {high) }}$ can be formulated as

$$
\bar{S}_{m ; n}^{(\mathrm{high})}=\frac{4 \delta_{m n}^{2} \delta_{m n^{\prime}}^{2}}{\delta_{m n}^{4}-\delta_{m n^{\prime}}^{4}}\left[U_{a}^{(m)}\left(\delta_{m n}\right)+\frac{V_{a}^{(m)}\left(\delta_{m n}\right)}{\sqrt{1-\delta_{m n}^{2}}}+\frac{\alpha_{m n^{\prime}} \delta_{m n^{\prime}}}{2 \delta_{m n}^{2} \beta}\left(\frac{1}{\sqrt{1+\delta_{m n}^{2}}}-\frac{1}{\sqrt{1-\delta_{m n}^{2}}}\right)\right],
$$

where

$$
\begin{aligned}
& U_{a}^{(m)}(b)=\frac{1}{\pi \beta} \sum_{r=0}^{N-1} \sum_{s=0}^{N-1}\left(\Omega^{(m)} a_{2 r+2 s+1}^{(m)}(b)+\lambda^{(m)} a_{2 r+2 s-1}^{(m)}(b)\right) \\
& V_{a}^{(m)}(b)=\frac{1}{4 b^{3} \beta} \sum_{r=0}^{N-1} \sum_{s=0}^{N-1}\left(\frac{\Delta^{(m)}+b^{2} \lambda^{(m)}}{b^{2 r+2 s+1}}\right), \\
& \Omega^{(m)}(r, s)=\alpha_{m n} \delta_{m n} \alpha_{m n^{\prime}} \delta_{m n^{\prime}} \hat{\gamma}_{m, m}(r, s)+\left(\alpha_{m n} \delta_{m n}+\alpha_{m n^{\prime}} \delta_{m n^{\prime}}\right) h_{m}(r) h_{m+1}(s), \\
& \lambda^{(m)}(r, s)=\hat{\gamma}_{m, m+1}(r, s)+\left(\alpha_{m n} \delta_{m n}+\alpha_{m n^{\prime}} \delta_{m n^{\prime}}\right) g_{m}(r) g_{m+1}(s),
\end{aligned}
$$

and the function $a_{n}^{(m)}(b)$ has been defined in App. A.

The asymptotic formulae for $S_{m ; n^{\prime}}$ have been obtained in the analogous way

$$
\begin{aligned}
& S_{m ; n^{\prime}}^{(\mathrm{high})}=\tilde{S}_{m ; n^{\prime}}+\bar{S}_{m ; n^{\prime}}^{(\mathrm{high})}, \\
& \tilde{S}_{m ; n^{\prime}}=\frac{2(-1)^{m} \delta_{m n}^{2} \delta_{m n^{\prime}}^{2}}{\sqrt{\pi} \beta^{3 / 2}\left(1-\delta_{m n^{\prime}}^{4}\right)\left(\delta_{m n}^{4}-\delta_{m n^{\prime}}^{4}\right)}\left[X_{a}^{(m)} \cos (2 \beta+\pi / 4)+Y_{a}^{(m)} \sin (2 \beta+\pi / 4)\right], \\
& \bar{S}_{m ; n^{\prime}}^{(\mathrm{high})}=\frac{4 \delta_{m n}^{2} \delta_{m n^{\prime}}^{2}}{\delta_{m n}^{4}-\delta_{m n^{\prime}}^{4}}\left[U_{a}^{(m)}\left(\delta_{m n^{\prime}}\right)+\frac{V_{a}^{(m)}\left(\delta_{m n^{\prime}}\right)}{\sqrt{1-\delta_{m n^{\prime}}^{2}}}+\frac{\alpha_{m n} \delta_{m n}}{2 \delta_{m n^{\prime}}^{2} \beta}\left(\frac{1}{\sqrt{1+\delta_{m n^{\prime}}^{2}}}-\frac{1}{\sqrt{1-\delta_{m n^{\prime}}^{2}}}\right)\right] .
\end{aligned}
$$

According to Eqs. (38) the asymptotic formula for the mutual resistance is

$$
\theta_{m ; n, n^{\prime}}^{(\text {high })}=S_{m ; n}^{(\text {high })}-S_{m ; n^{\prime}}^{(\text {high }} \text {. }
$$

Further, it has been obtained

$$
\theta_{m ; n, n^{\prime}}^{(\text {high })}=\tilde{\theta}_{m ; n, n^{\prime}}+\bar{S}_{m ; n}^{(\text {high })}-\bar{S}_{m ; n^{\prime}}^{(\text {high })}
$$

by employing (44), (47) and (49) where

$$
\tilde{\theta}_{m ; n, n^{\prime}}=\frac{2(-1)^{m} \delta_{m n}^{2} \delta_{m n^{\prime}}^{2}}{\sqrt{\pi} \beta^{3 / 2}\left(1-\delta_{m n}^{4}\right)\left(1-\delta_{m n^{\prime}}^{4}\right)}\left[X_{a}^{(m)} \cos (2 \beta+\pi / 4)+Y_{a}^{(m)} \sin (2 \beta+\pi / 4)\right] .
$$

The quantity $W_{m ; n}$ can be presented in the form of

$$
W_{m ; n}=\frac{4 \delta_{m n}^{2} \delta_{m n^{\prime}}^{2}}{\delta_{m n}^{4}-\delta_{m n^{\prime}}^{4}}\left[\alpha_{m n} \delta_{m n} \alpha_{m n^{\prime}} \delta_{m n^{\prime}} A_{p}^{(m)}-\left(\alpha_{m n} \delta_{m n}+\alpha_{m n^{\prime}} \delta_{m n^{\prime}}\right) B_{p}^{(m)}+C_{p}^{(m)}\right],
$$

where

$$
\begin{aligned}
& A_{p}^{(m)}=\int_{1}^{\infty} \frac{J_{m}^{2}(\beta x) x \mathrm{~d} x}{\left(x^{4}-\delta_{m n}^{4}\right) \sqrt{x^{2}-1}}, \quad B_{p}^{(m)}=\int_{1}^{\infty} \frac{J_{m}(\beta x) J_{m+1}(\beta x) x^{2} \mathrm{~d} x}{\left(x^{4}-\delta_{m n}^{4}\right) \sqrt{x^{2}-1}}, \\
& C_{p}^{(m)}=\int_{1}^{\infty} \frac{J_{m+1}^{2}(\beta x) x^{3} \mathrm{~d} x}{\left(x^{4}-\delta_{m n}^{4}\right) \sqrt{x^{2}-1}} .
\end{aligned}
$$

Calculations of these integrals have been performed by using of the expansion series from Eq. (24) and Eq. (43). The asymptotics can be formulated as

$$
W_{m ; n}^{(\text {high })}=\tilde{W}_{m ; n}+\bar{W}_{m ; n}^{(\text {high })}
$$

after separating the oscillating and non-oscillating components. The oscillating component is

$$
\tilde{W}_{m ; n}=\frac{2(-1)^{m} \delta_{m n}^{2} \delta_{m n^{\prime}}^{2}}{\sqrt{\pi} \beta^{3 / 2}\left(1-\delta_{m n}^{4}\right)\left(\delta_{m n}^{4}-\delta_{m n^{\prime}}^{4}\right)}\left[X_{p}^{(m)} \cos (2 \beta+\pi / 4)+Y_{p}^{(m)} \sin (2 \beta+\pi / 4)\right],
$$

where

$$
X_{p}^{(m)}=\alpha_{m n} \delta_{m n} \alpha_{m n^{\prime}} \delta_{m n^{\prime}} \nu_{m, m}(\beta)-\left(\alpha_{m n} \delta_{m n}+\alpha_{m n^{\prime}} \delta_{m n^{\prime}}\right) u_{m, m+1}(\beta)-\nu_{m+1, m+1}(\beta),
$$


$Y_{p}^{(m)}=-\alpha_{m n} \delta_{m n} \alpha_{m n^{\prime}} \delta_{m n^{\prime}} u_{m, m}(\beta)-\left(\alpha_{m n} \delta_{m n}+\alpha_{m n^{\prime}} \delta_{m n^{\prime}}\right) \nu_{m, m+1}(\beta)+u_{m+1, m+1}(\beta)$.

The non-oscillating component assumes the form of

$$
\bar{W}_{m n}=\frac{4 \delta_{m n}^{2} \delta_{m n^{\prime}}^{2}}{\delta_{m n}^{4}-\delta_{m n^{\prime}}^{4}}\left[U_{p}^{(m)}\left(\delta_{m n}\right)+\frac{V_{p}^{(m)}\left(\delta_{m n}\right)}{\sqrt{1-\delta_{m n}^{2}}} \arcsin \left(\delta_{m n}\right)\right]
$$

after applying Eqs. (A.5) where

$$
\begin{aligned}
& U_{p}^{(m)}(b)=\frac{1}{\pi \beta} \sum_{r=0}^{N-1} \sum_{s=0}^{N-1}\left[\hat{\phi}^{(m)}(r, s) a_{2 r+2 s}^{(m)}(b)+\alpha_{m n} \delta_{m n} \alpha_{m n^{\prime}} \delta_{m n^{\prime}} h_{m}(r) h_{m}(s) a_{2 r+2 s+2}^{(m)}(b)\right. \\
& \left.\quad+g_{m+1}(r) g_{m+1}(s) a_{2 r+2 s-2}^{(m)}(b)\right] \\
& V_{p}^{(m)}(b)=\frac{1}{2 \pi \beta} \sum_{r=0}^{N-1} \sum_{s=0}^{N-1} \frac{1}{b^{2 r+2 s+5}}\left[\hat{\phi}^{(m)}(r, s) b^{2}+\alpha_{m n} \delta_{m n} \alpha_{m n^{\prime}} \delta_{m n^{\prime}} h_{m}(r) h_{m}(s)+g_{m+1}(r) g_{m+1}(s) b^{4}\right], \\
& \hat{\phi}^{(m)}(r, s)=\alpha_{m n} \alpha_{m n^{\prime}} \delta_{m n} \delta_{m n^{\prime}} g_{m}(r) g_{m}(s)-\left(\alpha_{m n} \delta_{m n}+\alpha_{m n^{\prime}} \delta_{m n^{\prime}}\right) \hat{\gamma}_{m, m+1}(r, s)+h_{m+1}(r) h_{m+1}(s) .
\end{aligned}
$$

The asymptotic formula for $W_{m ; n^{\prime}}^{(\text {high }}$ can be found in a similar way giving

$$
\begin{aligned}
& W_{m ; n^{\prime}}^{(\text {high })}=\tilde{W}_{m ; n^{\prime}}+\bar{W}_{m ; n^{\prime}}^{(\mathrm{high})} \\
& \tilde{W}_{m ; n^{\prime}}=\frac{2(-1)^{m} \delta_{m n}^{2} \delta_{m n^{\prime}}^{2}}{\sqrt{\pi} \beta^{3 / 2}\left(1-\delta_{m n^{\prime}}^{4}\right)\left(\delta_{m n}^{4}-\delta_{m n^{\prime}}^{4}\right)}\left[X_{p}^{(m)} \cos (2 \beta+\pi / 4)+Y_{p}^{(m)} \sin (2 \beta+\pi / 4)\right] \\
& \bar{W}_{m ; n^{\prime}}^{(\mathrm{high})}=\frac{4 \delta_{m n}^{2} \delta_{m n^{\prime}}^{2}}{\delta_{m n}^{4}-\delta_{m n^{\prime}}^{4}}\left[U_{p}^{(m)}\left(\delta_{m n^{\prime}}\right)+\frac{V_{p}^{(m)}\left(\delta_{m n^{\prime}}\right)}{\sqrt{1-\delta_{m n^{\prime}}^{2}}} \arcsin \delta_{m n^{\prime}}\right]
\end{aligned}
$$

The asymptotic formulation for the mutual acoustic reactance can be formulated as

$$
\chi_{m ; n, n^{\prime}}^{(\text {high })}=W_{m ; n}^{(\text {high })}-W_{m ; n^{\prime}}^{(\text {high }}
$$

using Eq. (38). Further, using (54), (55) and (59) gives

$$
\chi_{m ; n, n^{\prime}}^{(\text {high) }}=\tilde{\chi}_{m ; n, n^{\prime}}+\bar{W}_{m ; n}^{(\text {high })}-\bar{W}_{m ; n^{\prime}}^{(\text {high })}
$$

where

$$
\tilde{\chi}_{m ; n, n^{\prime}}=\frac{2(-1)^{m} \delta_{m n}^{2} \delta_{m n^{\prime}}^{2}}{\sqrt{\pi} \beta^{3 / 2}\left(1-\delta_{m n}^{4}\right)\left(1-\delta_{m n^{\prime}}^{4}\right)}\left[X_{p}^{(m)} \cos (2 \beta+\pi / 4)+Y_{p}^{(m)} \sin (2 \beta+\pi / 4)\right] .
$$

The asymptotics valid within the high frequencies have been derived with the following absolute correction to their true values

$$
\left|\Delta \theta_{m ; n, n^{\prime}}^{(\mathrm{high})}\right|,\left|\Delta \chi_{m ; n, n^{\prime}}^{(\mathrm{high}}\right| \leq \delta_{m n}^{2} \delta_{m n^{\prime}}^{2} \beta^{-3 / 2}=E_{t}^{(\mathrm{high})} .
$$

This absolute correction to the true value decreases very rapidly with an increase in frequency (cf. the correction to the true values of the self impedance). The formulae obtained can be useful for numerical computations of the mutual acoustic impedance for $\beta>\beta_{m n}, \beta_{m n^{\prime}}$.

\subsection{The modal mutual impedance within the low frequencies}

The following conditions are satisfied $\delta_{m n}, \delta_{m n^{\prime}}>1$ within the low frequencies. In this case, the approximation for the mutual acoustic impedance can be directly derived from Eqs. (50) and (60) by using Eqs. (33). Finally, the acoustic mutual resistance and reactance has been formulated as

$$
\begin{aligned}
& \theta_{m ; n, n^{\prime}}^{(\text {low }}=\tilde{\theta}_{m ; n, n^{\prime}}+\bar{S}_{m ; n}^{(\text {low })}-\bar{S}_{m ; n^{\prime}}^{(\text {low })} \\
& \chi_{m ; n, n^{\prime}}^{(\text {low }}=\tilde{\chi}_{m ; n, n^{\prime}}+\bar{W}_{m ; n}^{(\text {low })}-\bar{W}_{m ; n^{\prime}}^{(\text {low })}
\end{aligned}
$$

respectively, where

$$
\begin{aligned}
& \bar{S}_{m ; n}^{\text {(low })}=\frac{4 \delta_{m n}^{2} \delta_{m n^{\prime}}^{2}}{\delta_{m n}^{4}-\delta_{m n^{\prime}}^{4}}\left[U_{a}^{(m)}\left(\delta_{m n}\right)-\frac{\pi V_{p}^{(m)}\left(\delta_{m n}\right)}{2 \sqrt{\delta_{m n}^{2}-1}}+\frac{\alpha_{m n^{\prime}} \delta_{m n^{\prime}}}{2 \delta_{m n}^{2} \beta \sqrt{1+\delta_{m n}^{2}}}\right], \\
& \bar{S}_{m ; n^{\prime}}^{\text {(low })}=\frac{4 \delta_{m n}^{2} \delta_{m n^{\prime}}^{2}}{\delta_{m n}^{4}-\delta_{m n^{\prime}}^{4}}\left[U_{a}^{(m)}\left(\delta_{m n^{\prime}}\right)-\frac{\pi V_{p}^{(m)}\left(\delta_{m n^{\prime}}\right)}{2 \sqrt{\delta_{m n^{\prime}}^{2}-1}}+\frac{\alpha_{m n} \delta_{m n}}{2 \delta_{m n^{\prime}}^{2} \beta \sqrt{1+\delta_{m n^{\prime}}^{2}}}\right],
\end{aligned}
$$




$$
\begin{aligned}
& \bar{W}_{m ; n}^{\text {(low })}=\frac{4 \delta_{m n}^{2} \delta_{m n^{\prime}}^{2}}{\delta_{m n}^{4}-\delta_{m n^{\prime}}^{4}}\left[U_{p}^{(m)}\left(\delta_{m n}\right)+\frac{V_{a}^{(m)}\left(\delta_{m n}\right)}{\sqrt{\delta_{m n}^{2}-1}}-\frac{\alpha_{m n^{\prime}} \delta_{m n^{\prime}}}{2 \delta_{m n}^{2} \beta \sqrt{\delta_{m n}^{2}-1}}-\frac{V_{p}^{(m)}\left(\delta_{m n}\right) \arccos h \delta_{m n}}{\sqrt{\delta_{m n}^{2},-1}}\right] \\
& \bar{W}_{m ; n^{\prime}}^{\text {(low) }}=\frac{4 \delta_{m n}^{2} \delta_{m n^{\prime}}^{2}}{\delta_{m n}^{4}-\delta_{m n^{\prime}}^{4}}\left[U_{p}^{(m)}\left(\delta_{m n^{\prime}}\right)+\frac{V_{a}^{(m)}\left(\delta_{m n^{\prime}}\right)}{\sqrt{\delta_{m n^{\prime}}^{2}-1}}-\frac{\alpha_{m n} \delta_{m n}}{2 \delta_{m n^{\prime}}^{2} \beta \sqrt{\delta_{m n^{\prime}}^{2}-1}}-\frac{V_{p}^{(m)}\left(\delta_{m n^{\prime}}\right) \arccos h \delta_{m n^{\prime}}}{\sqrt{\delta_{m n^{\prime}}^{2}-1}}\right] .
\end{aligned}
$$

The absolute correction to the true values of the self-resistance and the self-reactance can be estimated by the following expression [16]

$$
\left|\Delta \theta_{m ; n, n^{\prime}}^{\text {(low })}\right|,\left|\Delta \chi_{m ; n, n^{\prime}}^{\text {(low })}\right| \leq \delta_{m n}^{-2} \delta_{m n^{\prime}}^{-2} \beta^{-3 / 2}=E_{t}^{(\text {low })},
$$

within the low frequency range. The obtained formulae, like in the case of self impedance are valid for $7<\beta<$ $\beta_{m n}, \beta_{m n^{\prime}}$.

\subsection{The modal mutual impedance within the middle frequencies}

The middle frequency range can be defined by $\delta_{m n}<1<\delta_{m n^{\prime}}$. The approximate formulations for the modal mutual impedance can be obtained directly from the asymptotic formulae given in (44), (45), (47), (54), (55), (57) and (64) based on Eq. (38a)

$$
\begin{aligned}
& \theta_{m ; n, n^{\prime}}^{(\text {middle })}=S_{m ; n}^{(\text {high })}-S_{m ; n^{\prime}}^{(\text {low }}=\tilde{\theta}_{m ; n, n^{\prime}}+\bar{S}_{m ; n}^{(\text {high })}-\bar{S}_{m ; n^{\prime}}^{(\text {low }),} \\
& \chi_{m ; n, n^{\prime}}^{(m i d l l e)}=W_{m ; n}^{(\text {high })}-W_{m ; n^{\prime}}^{(\text {low }}=\tilde{\chi}_{m ; n, n^{\prime}}+\bar{W}_{m ; n}^{(\text {high })}-\bar{W}_{m ; n^{\prime}}^{(\text {low })},
\end{aligned}
$$

where the upper indice "middle" denotes that the expression is valid for the middle frequencies. The absolute correction to the true values of the modal resistance and reactance is [16]

$$
\left|\Delta \theta_{m ; n, n^{\prime}}^{(m i d l e)}\right|,\left|\Delta \chi_{m ; n, n^{\prime}}^{(m i d d l e)}\right| \leq \delta_{m n}^{2} \delta_{m n^{\prime}}^{-2} \beta^{-3 / 2}=E_{t}^{(\text {middle })}
$$

for the middle frequencies.

\section{Numerical analysis}

The modal resistance and reactance, and the corresponding absolute correction to their true values have been illustrated in Figs. 2-8. The quantities have been

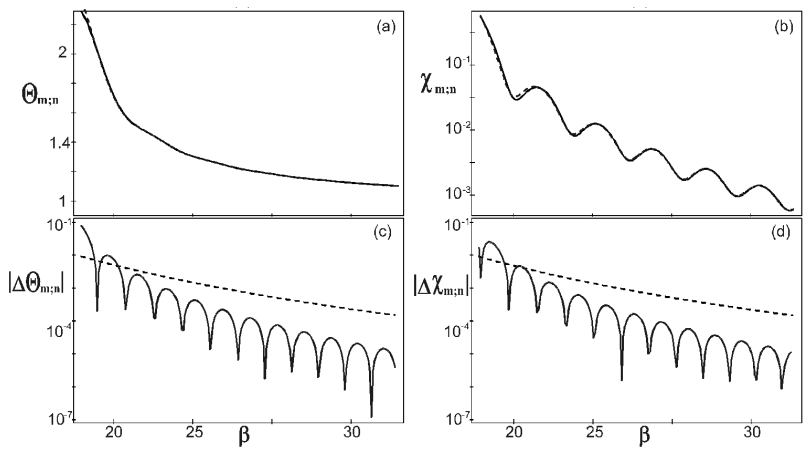

Fig. 2. The modal acoustic self impedance related to the mode $(11,1)$ within the high frequency range: (a) resistance, (b) reactance, (c) and (d) the corresponding absolute correction to their true values. Key: (a) and (b) solid line - the exact formula, dashed line - the asymptotic formula, (c) and (d) solid line-the correction estimation, dashed line - theoretical correction.

plotted using the integral formulae as well as the asymptotic and approximate formulations. The correction to their true value of a quantity $Q$ has been defined as [19]

$$
\Delta Q=Q_{\text {exact }}-\bar{Q},
$$

where $Q_{\text {exact }}$ and $\bar{Q}$ denote the true value of $Q$ obtained from the integral and its approximation or asymptotics,

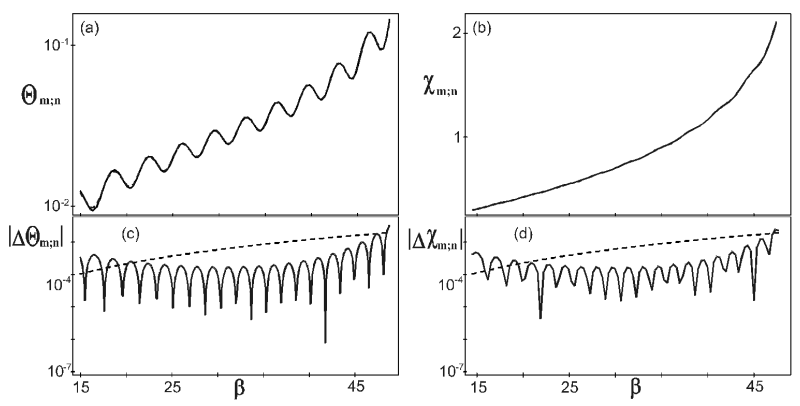

Fig. 3. The modal acoustic self impedance related to the mode $(11,12)$ within the low frequency range: (a) resistance, (b) reactance, (c) and (d) the corresponding absolute correction to their true values. Key: (a) and (b) solid line - the exact formula, dashed line - the asymptotic formula, (c) and (d) solid line-the correction estimation, dashed line - theoretical correction.

respectively. The absolute values of this correction have been shown in all the following figures where the logarithmic axes have been used for convenience. It is necessary for the impedances calculations to determine a suitable number $\mathrm{N}$ of terms in the asymptotic series from (10). The selection of the number $N$ depends on the desired accuracy and on the modal number $m$ (the number of the nodal diameters) of the interacting modes. The correction to the true value of considered quantities is not equal to zero. This fact results from using the stationary phase method and asymptotic expressions (12). The number of terms $N$ necessary to use in Eq. (12) has been 

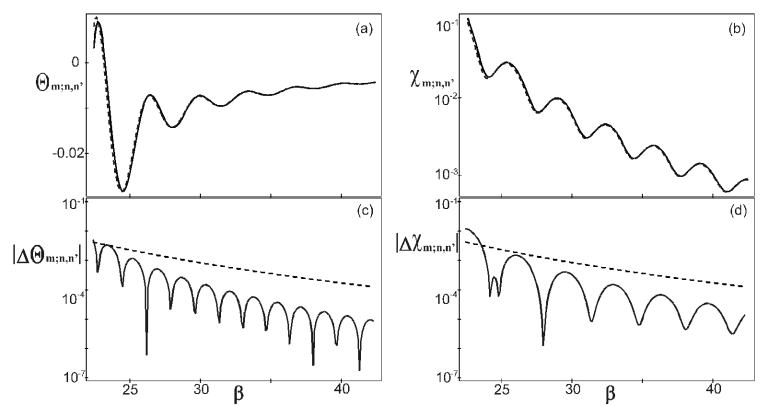

Fig. 4. The modal acoustic mutual impedance related to the mode $(11,1)$ and $(11,2)$ within the high frequency range: (a) resistance, (b) reactance, (c) and (d) the corresponding absolute correction to their true values. Key: (a) and (b) solid line - the exact formula, dashed line - the asymptotic formula, (c) and (d) solid line-the correction estimation, dashed line - theoretical correction.

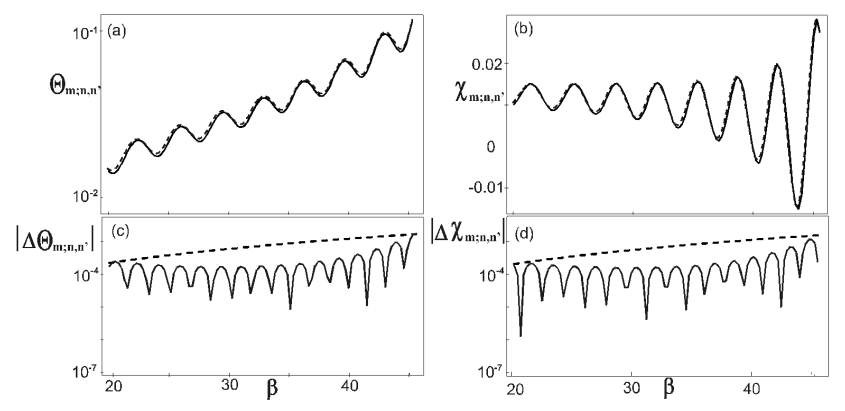

Fig. 5. The modal acoustic mutual impedance related to the mode $(11,11)$ and $(11,12)$ within the low frequency range: (a) resistance, (b) reactance, (c) and (d) the corresponding absolute correction to their true values. Key: (a) and (b) solid line - the exact formula, dashed line - the asymptotic formula, (c) and (d) solid line-the correction estimation, dashed line - theoretical correction.

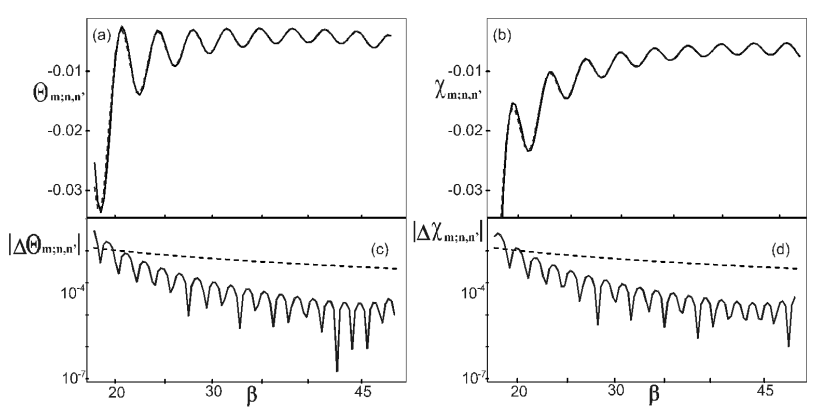

Fig. 6. The modal acoustic mutual impedance related to the mode $(11,1)$ and $(11,12)$ within the middle frequency range: (a) resistance, (b) reactance, (c) and (d) the corresponding absolute correction to their true values. Key: (a) and (b) solid line - the exact formula, dashed line - the asymptotic formula, (c) and (d) solid line-the correction estimation, dashed line - theoretical correction.

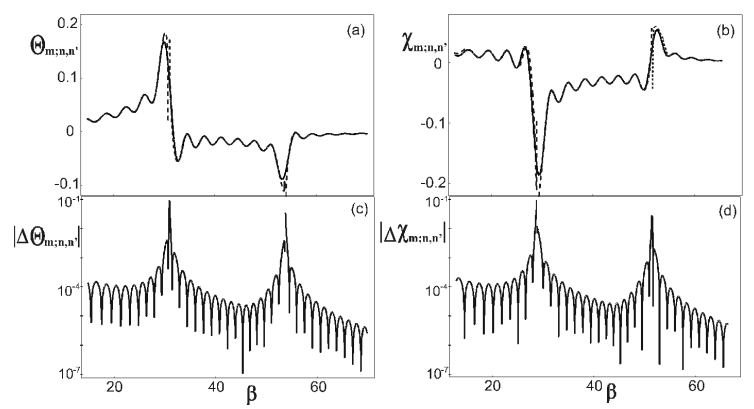

Fig. 7. The modal acoustic mutual impedance related to the mode $(11,5)$ and $(11,12)$ within the high frequency range containing both resonance frequencies: (a) resistance, (b) reactance, (c)-(d) the corresponding absolute correction to their true values. Key: (a)-(b) solid line - the exact formula, dashed line - the asymptotic formula.

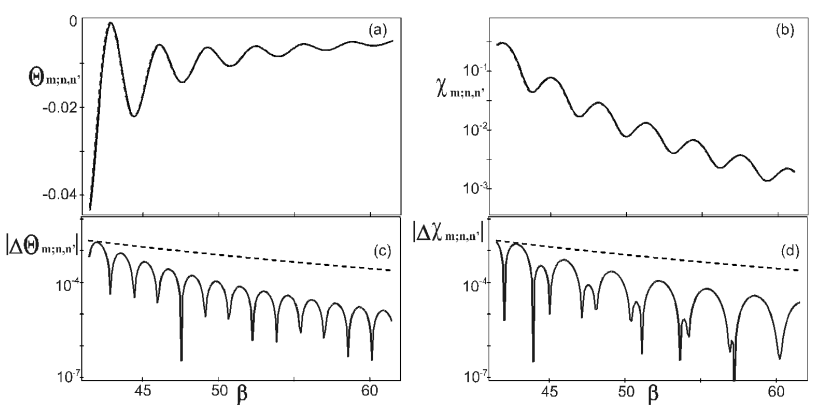

Fig. 8. The modal acoustic mutual impedance related to the pair of modes $(0,11)$ and $(0,12)$, and the high frequencies: (a) resistance, (b) reactance, (c)-(d) the corresponding absolute correction to their true values. Key: (a)-(b) solid line - exact formula, dashed line-asymptotic formula, (c)-(d) solid line-correction estimation, dashed line - theoretical correction.

obtained empirically by plotting a number of curves with different values of $N$. This value is

$$
N=\left\lfloor\frac{m+1}{2}\right\rfloor,
$$

where $\lfloor x\rfloor$ denotes the greatest integer smaller or equal to $x$. As the consequence, the estimate absolute correction to the true value does not exceed its theoretical value within all the considered frequency bands.

The presented asymptotic formulae are useful for the calculations of the self-impedance and the mutual-impedance within the high frequency range. It is obvious that accuracy of this formulae grows rapidly with an increase in $\beta$. The self-resistance and the self-reactance related to the mode $(11,1)$ can be calculated with the absolute correction to the true value smaller than $10^{-3}$ by assuming $\beta>23$, (Fig. 2). The absolute correction to the true value for the mutual resistance and the mutual reactance do not exceed $10^{-3}$ for the mode pair $(11,1)$ and $(11,2)$ when $\beta>28$ (Fig. 4). Figures 3 and 5 show that for the lower vibrations frequencies the approximation 
accuracy decreases when the frequency tends to the resonance value. The absolute correction to the true value increase is also observed for very low frequencies. That fact results from using the special functions asymptotics which are less accurate as the $\beta$ parameter decreases. The self resistance and the self reactance have been computed with the absolute correction to the true value smaller than $10^{-3}$ for $\beta \in(15,45)$ in the case of mode $(11,12)$ within the low frequency range (Fig. 3). Based on an analysis of Fig. 5, it is confirmed that the reached approximate expression enables the calculations of the mutual resistance and the mutual reactance with the absolute correction to the true value smaller than $10^{-3}$ for $\beta \in(20,45)$ in the case of pair of the modes $(11,11)$, $(11,12)$. For low frequencies the absolute correction to the true value is always greater than $10^{-5}$ for all considered modes. The absolute correction to the true value for the mutual resistance and reactance in the case of the mode pairs $(11,1),(11,12)$ is smaller than $10^{-4}$ for almost the whole range of the middle frequencies. Only for some close to coincidence frequencies, the absolute correction to the true value assumes some great values (Fig. 6).

It is possible to determine the frequency bands about the corresponding coincidence frequencies for which the formulae presented are useless with regard to an increase in the correction to their true value (Fig. 7). It can be noticed how narrow are the frequency bands about the coincidence frequencies, where the absolute correction to their true value exceeds $10^{-3}$. The width of the bands of $k_{0} a$ is not greater than 7 . The formulae obtained are useless within these narrow bands and the integral formulae must be used instead.
The high frequency asymptotics obtained are more accurate than the approximate expressions appropriate to the low and middle frequencies. The required accuracy of the asymptotics valid for the high frequencies can be achieved by increasing the $\beta$ parameter value. In the case of low and middle frequencies, the absolute correction to their true values approaches its minimal value.

The approximate and asymptotic formulae achieved are also useful in the particular case of axisymmetric modes, i.e. for $m=0$. As an example, the mutual-resistance and the mutual-reactance related to the pair of modes $(0,11)$ and $(0,12)$ together with the corresponding absolute correction to their true values (Fig. 8). The absolute correction in this case is smaller than $10^{-3}$ when $\beta>45$.

\section{Conclusions}

The approximate and asymptotic formulae of the asymmetric self impedance and the mutual impedance of a vibrating clamped circular plate embedded in a flat rigid baffle have been obtained. The formulation obtained enable calculations within almost the whole frequency range for $\beta>7$, except the frequencies close to the coincidence. They are sufficiently accurate both for some axisymmetric and asymmetric modes. In the case of the asymmetric vibrations, the accuracy growth has been reached by taking into account the proper number of terms to approximate the special function products. The elementary form of the formulae obtained is useful for numerical analysis.

\section{Appendix A. The exact values of the integrals}

In order to present the asymptotics for the self and mutual impedance in an elementary form, it is necessary to calculate the following integrals

$$
I_{n}^{(s)}(b)=\int_{1}^{\infty} \frac{\mathrm{d} x}{x^{n}\left(x^{4}-b^{4}\right)^{2} \sqrt{x^{2}-1}}, \quad I_{n}^{(m)}(b)=\int_{1}^{\infty} \frac{\mathrm{d} x}{x^{n}\left(x^{4}-b^{4}\right) \sqrt{x^{2}-1}}
$$

for $n=-2,-1,0, \ldots$, where it has been assumed that $b<1$.

The above integrals can be transformed into the following form

$$
\begin{aligned}
& I_{n}^{(s)}(b)=\frac{1}{4 b^{2}}\left[\frac{1}{4 b^{3}}\left(\hat{I}_{n+2}^{(a)}(b)-\hat{I}_{n+2}^{(b)}(b)+b \hat{I}_{n+2}^{(c)}(b)+d \hat{I}_{n+2}^{(d)}(b)\right)-\hat{I}_{n+2}^{(f)}(b)\right], \\
& I_{n}^{(m)}(b)=\frac{1}{4 b}\left(\hat{I}_{n+2}^{(b)}(b)-\hat{I}_{n+2}^{(a)}(b)\right)+\frac{1}{2} \hat{I}_{n+2}^{(e)}(b),
\end{aligned}
$$

where

$$
\begin{array}{ll}
\hat{I}_{n}^{(a)}(b)=\int_{1}^{\infty} \frac{\mathrm{d} x}{x^{n}(x+b) \sqrt{x^{2}-1}}, \quad \hat{I}_{n}^{(b)}(b)=\int_{1}^{\infty} \frac{\mathrm{d} x}{x^{n}(x-b) \sqrt{x^{2}-1}}, \\
\hat{I}_{n}^{(c)}(b)=\int_{1}^{\infty} \frac{\mathrm{d} x}{x^{n}(x+b)^{2} \sqrt{x^{2}-1}}, \quad \hat{I}_{n}^{(\mathrm{d})}(b)=\int_{1}^{\infty} \frac{\mathrm{d} x}{x^{n}(x-b)^{2} \sqrt{x^{2}-1}}, \\
\hat{I}_{n}^{(e)}(b)=\int_{1}^{\infty} \frac{\mathrm{d} x}{x^{n}\left(x^{2}+b^{2}\right) \sqrt{x^{2}-1}}, \quad \hat{I}_{n}^{(f)}(b)=\int_{1}^{\infty} \frac{\mathrm{d} x}{x^{n}\left(x^{2}+b^{2}\right)^{2} \sqrt{x^{2}-1}},
\end{array}
$$

Integrals from Eq. (A.3) for $n=-2,-1,0, \ldots$ can be expressed as 


$$
\begin{aligned}
& \hat{I}_{n}^{(a)}(b)=-\sum_{s=0}^{n-1}\left(-\frac{1}{b}\right)^{s+1} v_{n-s}+\left(-\frac{1}{b}\right)^{n} \frac{\arccos (b)}{\sqrt{1-b^{2}}}, \\
& \hat{I}_{n}^{(b)}(b)=-\sum_{s=0}^{n-1}\left(\frac{1}{b}\right)^{s+1} v_{n-s}+\left(\frac{1}{b}\right)^{n} \frac{\pi / 2+\arcsin (b)}{\sqrt{1-b^{2}}}, \\
& \hat{I}_{n}^{(c)}(b)=\sum_{s=0}^{n-1} \sum_{r=0}^{n-s-1}\left(-\frac{1}{b}\right)^{s+r+2} v_{n-s-r}+\frac{(-1)^{n}}{b^{n}\left(1-b^{2}\right)}+\frac{(-1)^{n}\left(n-b^{2}(n+1)\right)}{b^{n+1}\left(1-b^{2}\right)^{3 / 2}} \arccos (b), \\
& \hat{I}_{n}^{(d)}(b)=\sum_{s=0}^{n-1} \sum_{r=0}^{n-s-1}\left(\frac{1}{b}\right)^{s+r+2} v_{n-s-r}+\frac{1}{b^{n}\left(1-b^{2}\right)}-\frac{\left(n-b^{2}(n+1)\right)}{b^{n+1}\left(1-b^{2}\right)^{3 / 2}}(\pi / 2+\arcsin (b)), \\
& \hat{I}_{n}^{(e)}(b)=\left\{\begin{array}{ll}
\frac{\pi}{2 \sqrt{1+b^{2}}} & \text { for } n=-1 \\
\frac{\operatorname{arcsinh}(b)}{b \sqrt{1+b^{2}}} & \text { for } n=0 \\
\frac{1}{b^{2}}\left(v_{n}-\hat{I}_{n-2}^{(e)}(b)\right) & \text { for } n>0
\end{array},\right. \\
& \hat{I}_{n}^{(f)}(b)=\left\{\begin{array}{ll}
\frac{\pi}{4\left(1+b^{2}\right)^{3 / 2}} & \text { for } n=-1 \\
-\frac{1}{2 b^{2}\left(1+b^{2}\right)}\left[1-\left(1+2 b^{2}\right) \hat{I}_{0}^{(e)}(b)\right] & \text { for } n=0 \\
\frac{1}{b^{2}}\left(\hat{I}_{n}^{(e)}(b)-\hat{I}_{n-2}^{(f)}(b)\right) & \text { for } n>0
\end{array} \quad, \quad v_{n}= \begin{cases}\pi / 2 & \text { for } n=1 \\
1 & \text { for } n=2 . \\
\frac{(n-2)}{(n-1)} v_{n-2} & \text { for } n>2\end{cases} \right.
\end{aligned}
$$

Inserting expressions (A.4) into Eq. (A.2) provides

$$
I_{n}^{(m)}(b)=a_{n}^{(m)}(b)+\frac{1}{4 b^{n+3}} f_{n}(b), \quad I_{n}^{(s)}(b)=a_{n}^{(s)}(b)+b_{n}^{(s)}(b) f_{n}(b),
$$

where

$$
\begin{aligned}
& a_{n}^{(m)}(b)=-\frac{1}{4 b^{2}} \sum_{i=0}^{n+1} \frac{1+(-1)^{i}}{b^{i}} v_{n+2-i}+\frac{1}{2} \hat{I}_{n+2}^{(e)}(b), \\
& f_{n}(b)=\frac{\left[1-(-1)^{n}\right] \pi / 2+\left[1+(-1)^{n}\right] \arcsin (b)}{\sqrt{1-b^{2}}}, \\
& a_{n}^{(s)}(b)=\frac{1}{16 b^{6}}\left[\sum_{i=0}^{n+1} \frac{1+(-1)^{i}}{b^{i}} v_{n+2-i}+\sum_{i=0}^{n+1} \sum_{r=0}^{n-i+1} \frac{1+(-1)^{i+r}}{b^{i+r}} v_{n+2-i-r}+\frac{1+(-1)^{n}}{b^{n}\left(1-b^{2}\right)}\right]-\frac{1}{4 b^{2}} \hat{I}_{n+2}^{(f)}(b), \\
& b_{n}^{(s)}(b)=-\frac{n+3-b^{2}(n+4)}{16 b^{n+7}\left(1-b^{2}\right)} .
\end{aligned}
$$

Formulae (A.5) together with denotations (A.6) have been used to express some asymptotic expressions for modal acoustic impedance in a convenient for numerical calculation form.

\section{Appendix B. The total acoustic impedance and the coupling matrix}

The vibration amplitude can be presented as the complete eigenfunction system series

$$
W\left(r_{0}, \varphi_{0}\right)=\sum_{m=0}^{\infty} \sum_{n=1}^{\infty}\left[c_{m n}^{(c)} W_{m n}^{(c)}\left(r_{0}, \varphi_{0}\right)+c_{m n}^{(s)} W_{m n}^{(s)}\left(r_{0}, \varphi_{0}\right)\right],
$$

where

$$
\left\{\begin{array}{l}
W_{m n}^{(c)}\left(r_{0}, \varphi_{0}\right) \\
W_{m n}^{(s)}\left(r_{0}, \varphi_{0}\right)
\end{array}\right\}=A_{m n}\left[J_{m}\left(k_{m n} r_{0}\right)+B_{m n} I_{m}\left(k_{m n} r_{0}\right)\right]\left\{\begin{array}{c}
\cos m \varphi_{0} \\
\sin m \varphi_{0}
\end{array}\right\}
$$

are the corresponding eigenfunctions, $r_{0}, \varphi_{0}$ are plate's point polar coordinates, $k_{m n}^{2}=\omega_{m n} \sqrt{\rho h / D}$ is the structural wave number of the mode $(m, n), \omega_{m n}$ is the eigenfrequency, $\rho$ is the plate's density, $h$ is plate's thickness, $D=$ $E h^{3} /\left(12\left(1-\nu^{2}\right)\right)$ denotes the plate's stiffness, $E$ is Young modulus, $\nu$ is the Poisson ratio.

The normalized acoustic impedance can be formulated in the form of the infinite triple series

$$
\zeta=\theta-i \chi=\sum_{m=0}^{\infty} \sum_{n=1}^{\infty} \sum_{n^{\prime}=1}^{\infty}\left\{\tilde{c}_{m, n}^{(c)} \tilde{c}_{m, n}^{(c) *}+\operatorname{sign}_{m} \tilde{c}_{m, n}^{(s)} \tilde{c}_{m, n}^{(s) *}\right\} \zeta_{m ; n, n^{\prime}}
$$

where $\theta, \chi$ denote resistance and reactance, respectively, 


$$
\begin{aligned}
& \tilde{c}_{m, n}^{(c)}=\frac{\omega}{\sqrt{2\left\langle\left|v_{\mathrm{N}}\right|^{2}\right\rangle}} c_{m, n}^{(c)}, \quad \tilde{c}_{m, n}^{(s)}=\frac{\omega}{\sqrt{2\left\langle\left|v_{\mathrm{N}}\right|^{2}\right\rangle}} c_{m, n}^{(s)}, \\
& \tilde{c}_{m, n}^{(c) *}=\frac{\omega}{\sqrt{2\left\langle\left|v_{\mathrm{N}}\right|^{2}\right\rangle}} c_{m, n}^{(c) *}, \quad \tilde{c}_{m, n}^{(s) *}=\frac{\omega}{\sqrt{2\left\langle\left|v_{\mathrm{N}}\right|^{2}\right\rangle}} c_{m, n}^{(s) *}, \\
& \left\langle\left|v_{\mathrm{N}}\right|^{2}\right\rangle=\frac{1}{2 S} \int_{\mathrm{S}} v_{\mathrm{N}}(\boldsymbol{r}) v_{\mathrm{N}}^{*}(\boldsymbol{r}) \mathrm{d} S=\frac{\omega^{2}}{2} \sum_{m=0}^{\infty} \sum_{n=1}^{\infty}\left\{\left|c_{m, n}^{(c)}\right|^{2}+\operatorname{sign}_{m}\left|c_{m, n}^{(s)}\right|^{2}\right\},
\end{aligned}
$$

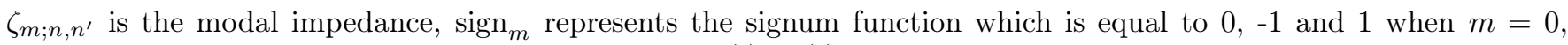
$m<0$ and $m>0$, respectively. The coupling factors $c_{m, n}^{(c)}, c_{m, n}^{(s)}$ can be obtained by solving of the equation of motion. In the case when the acoustic attenuation is neglected the normalized constants $c_{m, n}^{(c)}, c_{m, n}^{(s)}$ can be expressed in the form of the following coupling matrix

$$
\left\{\begin{array}{l}
c_{m, n}^{(c)} \\
c_{m, n}^{(s)}
\end{array}\right\}=\frac{1}{\omega^{2} \rho h\left(k_{\mathrm{D}}^{-4} k_{m, n}^{4}-1\right)}\left\{\begin{array}{l}
f_{m, n}^{(c)} \\
f_{m, n}^{(s)}
\end{array}\right\},
$$

where

$$
\left\{\begin{array}{l}
f_{m, n}^{(c)} \\
f_{m, n}^{(s)}
\end{array}\right\}=\frac{1}{S} \int_{\mathrm{S}}\left\{\begin{array}{l}
W_{m, n}^{(c)}(\boldsymbol{r}) \\
\operatorname{sign}_{m} W_{m, n}^{(s)}(\boldsymbol{r})
\end{array}\right\} f(\boldsymbol{r}) \mathrm{d} S
$$

are the excitation factors, $\omega$ is the excitation frequency, $f(\boldsymbol{r})$ is the excitation, $k_{\mathrm{D}}^{-4}=D / \rho h \omega^{2}$.

Equation (B.2) represents the exact value. However, in practical applications only its approximation can be obtained on the basis of Eqs. (B.2) and (B.4). Limiting the infinite sum to the finite number of terms results in the following asymptotic formulae

$$
\theta=\theta_{1}+\Delta \theta_{1}, \quad \chi=\chi_{1}+\Delta \chi_{1},
$$

where

$$
\begin{aligned}
& \theta_{1}=\sum_{m=0}^{\mathrm{M}-1} \sum_{n=1}^{N-1} \sum_{n^{\prime}=1}^{N-1}\left\{\tilde{c}_{m, n}^{(c)} \tilde{c}_{m, n}^{(c) *}+\operatorname{sign}_{m} \tilde{c}_{m, n}^{(s)} \tilde{c}_{m, n}^{(s) *}\right\} \theta_{m ; n, n^{\prime}}, \\
& \chi_{1}=\sum_{m=0}^{\mathrm{M}-1} \sum_{n=1}^{N-1} \sum_{n^{\prime}=1}^{N-1}\left\{\tilde{c}_{m, n}^{(c)} \tilde{c}_{m, n}^{(c) *}+\operatorname{sign}_{m} \tilde{c}_{m, n}^{(s)} \tilde{c}_{m, n}^{(s) *}\right\} \chi_{m ; n, n^{\prime}} .
\end{aligned}
$$

Further, it is convenient to introduce its relative approximation error as follows

$$
\delta \theta \leq \frac{\left|\Delta \theta_{1}\right|+\left|\Delta \theta_{2}\right|}{\theta}, \quad \delta \chi \leq \frac{\left|\Delta \chi_{1}\right|+\left|\Delta \chi_{2}\right|}{\chi},
$$

where $\left|\Delta \theta_{1}\right|$ and $\left|\Delta \chi_{1}\right|$ are the absolute approximation errors of the quantities given in Eq. (B.2) caused by limiting the infinite series to the finite number of terms used, and $\left|\Delta \theta_{2}\right|$ and $\left|\Delta \chi_{2}\right|$ are the absolute approximate errors of the same quantities caused by the approximation of the corresponding modal quantities. The former approximation errors can be estimated as follows

$$
\begin{aligned}
& \Delta \theta_{1} \leq \sum_{m=0}^{\mathrm{M}} \sum_{n=1}^{N}\left\{\bar{c}_{m, n}^{(c)} \bar{c}_{m, N}^{(c) *}+\operatorname{sign}_{m} \bar{c}_{m, n}^{(s)} \bar{c}_{m, N}^{(s) *}\right\} \theta_{m ; n, N}+\sum_{m=0}^{\mathrm{M}} \sum_{n^{\prime}=1}^{N}\left\{\bar{c}_{m, N}^{(c)} \bar{c}_{m, n^{\prime}}^{(c) *}+\operatorname{sign}_{m} \bar{c}_{m, N}^{(s)} \bar{c}_{m, n^{\prime}}^{(s) *}\right\} \theta_{m ; N, n^{\prime}} \\
& \quad+\sum_{n, n^{\prime}=1}^{N-1}\left\{\bar{c}_{\mathrm{M}, \mathrm{n}}^{(c)} \bar{c}_{\mathrm{M}, \mathrm{n}^{\prime}}^{(c) *}+\operatorname{sign}_{\mathrm{M}} \bar{c}_{\mathrm{M}, \mathrm{n}}^{(s)} \bar{c}_{\mathrm{M}, \mathrm{n}^{\prime}}^{(s) *}\right\} \theta_{\mathrm{M} ; \mathrm{n}, \mathrm{n}^{\prime}}, \\
& \Delta \chi_{1} \leq \sum_{m=0}^{\mathrm{M}} \sum_{n=1}^{N}\left\{\bar{c}_{m, n}^{(c)} \bar{c}_{m, N}^{(c) *}+\operatorname{sign}_{m} \bar{c}_{m, n}^{(s)} \bar{c}_{m, N}^{(s) *}\right\} \chi_{m ; n, N}+\sum_{m=0}^{\mathrm{M}} \sum_{n^{\prime}=1}^{N}\left\{\bar{c}_{m, N}^{(c)} \bar{c}_{m, n^{\prime}}^{(c) *}+\operatorname{sign}_{m} \bar{c}_{m, N}^{(s)} \bar{c}_{m, n^{\prime}}^{(s) *}\right\} \chi_{m ; N, n^{\prime}} \\
& \quad+\sum_{n, n^{\prime}=1}^{N-1}\left\{\bar{c}_{\mathrm{M}, \mathrm{n}}^{(c)} \bar{c}_{\mathrm{M}, \mathrm{n}^{\prime}}^{(c) *}+\operatorname{sign}_{\mathrm{M}} \bar{c}_{\mathrm{M}, \mathrm{n}}^{(s)} \bar{c}_{\mathrm{M}, \mathrm{n}^{\prime}}^{(s) *}\right\} \chi_{\mathrm{M} ; \mathrm{n}, \mathrm{n}^{\prime}},
\end{aligned}
$$

and the later as

$$
\Delta \theta_{2}=\sum_{m=0}^{\mathrm{M}-1} \sum_{n=1}^{N-1} \sum_{n^{\prime}=1}^{N-1}\left\{\tilde{c}_{m, n}^{(c)} \tilde{c}_{m, n}^{(c) *}+\operatorname{sign}_{m} \tilde{c}_{m, n}^{(s)} \tilde{c}_{m, n}^{(s) *}\right\} \Delta \theta_{m ; n, n^{\prime}}
$$




$$
\Delta \chi_{2}=\sum_{m=0}^{\mathrm{M}-1} \sum_{n=1}^{N-1} \sum_{n^{\prime}=1}^{N-1}\left\{\tilde{c}_{m, n}^{(c)} \tilde{c}_{m, n}^{(c) *}+\operatorname{sign}_{m} \tilde{c}_{m, n}^{(s)} \tilde{c}_{m, n}^{(s) *}\right\} \Delta \chi_{m ; n, n^{\prime}},
$$

where $\Delta \theta_{m ; n, n^{\prime}}$ and $\Delta \chi_{m ; n, n^{\prime}}$ are the corrections to the true values of the modal resistance and the modal reactance. The corrections have been defined in Eq. (68) and this paper focuses on them.

\section{References}

[1] A. Berry, J.L. Guyader, J. Nicolas, Journal of the Acoustical Society of America 86, 2792 (1990).

[2] H. Lee, R. Singh, Noise Control Eng. J. 52 (5), 225 (2004).

[3] M. Amabili, G. Frosali, M.K. Kwak, Journal of Sound and Vibration 191, 825 (1996).

[4] J.H. Ginsberg, P. Chu, Journal of the Acoustical Society of America 91, 894 (1992).

[5] S. Alper, E.B. Magrab, Journal of the Acoustical Society of America 48, 681 (1970).

[6] X.-F. Wu, A.D. Pierce, J.H. Ginsberg, IEEE Journal of Oceanic Engineering OE-12, 412 (1987).

[7] H. Lee, R. Singh, Journal of Sound and Vibration 285, 1210 (2005), Short Communication.

[8] Z. Dingguo, M.J. Crocker, Archives of Acoustics 34, 25 (2009).

[9] Z. Dingguo, M.J. Crocker, Archives of Acoustics 34, 13 (2009).

[10] H. Levine, F.G. Leppington, Journal of Sound and Vibration 121, 269 (1988).

[11] C. Kauffmann, Journal of the Acoustical Society of America 104, 3245 (1998).
[12] L. Shuyu, Acta Acustica united with Acustica 86, 388 (2000).

[13] W.P. Rdzanek, W. Rdzanek, K. Szemela, Archives of Acoustics 34, 75 (2009).

[14] W.P. Rdzanek, W. Rdzanek, Z. Engel, K. Szemela, International Journal of Occupational Safety and Ergonomics 13, 147 (2007).

[15] J.P. Arenas, International Journal of Occupational Safety and Ergonomics 15, 401 (2009).

[16] W.P. Rdzanek, W.J. Rdzanek, K. Szemela, to be published in Journal of Computational Acoustics.

[17] P. Witkowski, Archives of Acoustics 22, 463 (1997).

[18] P.M. Morse, K.U. Ingard, Theoretical acoustics, McGraw-Hill, Inc. (1968).

[19] M. Abramowitz, I.A. Stegun, Ed. Handbook of mathematical functions with formulae, graphs, and mathematical tables, Applied Mathematics Series 55. U.S. Dept. of Commerce, National Bureau of Standards (1972).

[20] G.N. Watson, A treatise on the theory of Bessel functions, Cambridge University Press (1944). 